\title{
Open-Label Randomized Trial of Early Clinical Outcomes of Ceftaroline Fosamil Versus Vancomycin for the Treatment of Acute Bacterial Skin and Skin Structure Infections at Risk of Methicillin-Resistant Staphylococcus aureus
}

Kimberly C. Claeys · Evan J. Zasowski · Trang D. Trinh · Anthony M. Casapao · Jason M. Pogue $\cdot$ Nitin Bhatia Ryan P. Mynatt - Suprat S. Wilson - Crystal Arthur - Robert Welch • Robert Sherwin • Wasif Hafeez • Donald P. Levine · Keith S. Kaye · George Delgado • Christopher A. Giuliano • Robert Takla • Colleen Rieck • Leonard B. Johnson · Kyle P. Murray · James Gordon · Kate Reyes · Pamela Hartman · Susan L. Davis • Michael J. Rybak

\begin{abstract}
Introduction: Acute bacterial skin and skin structure infections (ABSSSIs) remain among the most common infectious processes seen in the clinical setting. For patients with complicated ABSSSIs deemed to require intravenous antibiotics, vancomycin remains the mainstay therapy. Ceftaroline has been shown to be non-
\end{abstract}

Enhanced digital features To view enhanced digital features for this article go to https://doi.org/10.6084/ m9.figshare.7825091.

K. C. Claeys · E. J. Zasowski · T. D. Trinh · A. M. Casapao - C. A. Giuliano - S. L. Davis . M. J. Rybak ( $\square)$

Anti-Infective Research Laboratory, Wayne State University Eugene Applebaum College of Pharmacy and Health Sciences, Detroit, MI, USA

e-mail: aa1592@wayne.edu

K. C. Claeys

Department of Pharmacy Practice and Science, University of Maryland School of Pharmacy,

Baltimore, MD, USA

A. M. Casapao

Department of Pharmacotherapy and Translational Research, University of Florida College of Pharmacy, Jacksonville, FL, USA inferior to vancomycin and may result in faster resolution of signs of infection.

Methods: Multicenter, prospective, open-label, randomized trial of ceftaroline versus vancomycin for the treatment of adult patients admitted for management of ABSSSIs from April 2012 to May 2016; 166 patients in the clinically evaluable (CE) group were needed to determine a $20 \%$ difference in primary outcome of clinical response at day 2 or 3 of antibiotics. Clinical response was defined as cessation of spread of lesion and improvement in systemic signs/ symptoms of infection. A secondary outcome

J. M. Pogue - N. Bhatia · R. P. Mynatt .

S. S. Wilson - C. Arthur · R. Welch - R. Sherwin . W. Hafeez - D. P. Levine · K. P. Murray · J. Gordon · M. J. Rybak

Detroit Medical Center, Detroit, MI, USA

\section{K. S. Kaye}

University of Michigan Medical School, Ann Arbor, MI, USA

G. Delgado - C. A. Giuliano - R. Takla · C. Rieck ·

L. B. Johnson

St. John Hospital and Medical Center, Detroit, MI, USA

K. Reyes · P. Hartman · S. L. Davis

Henry Ford Health System, Detroit, MI, USA 
was a $\geq 20 \%$ reduction in lesion size at day 2 or 3 of antibiotics.

Results: One hundred seventy-four patients were enrolled in the intention-to-treat (ITT) group and 108 were CE. Among CE patients, 54 were randomized to ceftaroline and 54 to vancomycin. Baseline characteristics were similar except patients in the ceftaroline arm were older and had a non-significantly higher degree of comorbidities (median Charlson score 2 vs. 4 , respectively). Cellulitis was the most common type of ABSSSI (85.2\% vs. $79.6 \%$, respectively). Rapid diagnostic testing of available cultures $(n=55)$ demonstrated high agreement with clinical microbiology for identification of Staphylococcus aureus (100\%) and MRSA (100\%). There was no significant difference in primary outcome of day 2 or 3 clinical response $(50.0 \%$ vs. $51.9 \%)$.

Conclusion: Early clinical response between vancomycin- and ceftaroline-treated ABSSSIs was similar. Patients with ABSSSIs rarely remained hospitalized for $>2-3$ days, thus limiting our ability to critically assess clinical outcomes.

Trial Registration: ClinicalTrials.gov identifier, NCT02582203.

Funding: Allergan plc.

Keywords: Acute bacterial skin and skin structure infection; Ceftaroline; Methicillinresistant $S$. aureus; Vancomycin

\section{INTRODUCTION}

Acute bacterial skin and skin structure infections (ABSSSIs) are among the most common infections encountered in the hospital setting [1-5]. There are approximately $600,000-800,000$ hospitalizations secondary to ABSSSIs per year, accounting for $2 \%$ of all hospital admissions [5]. The majority of these infections are treated in the emergency department, with abscess and cellulitis being reported as the primary infection [4]. Eldelsberg et al. [2], however, reported that national ABSSSI hospitalizations increased by 29\% from 2000 to 2004 . This is particularly significant as hospitalization for ABSSSI represents a significant cost burden to the US healthcare system, resulting in approximately $\$ 6$ billion per year in inpatient costs with the average patient stay estimated to cost $\$ 8000$ USD per patient. Itani et al. [6] identified 5156 patient cases of ABSSSIs from 2002 to 2006 for which the average length of hospital stay and cost of treatment was 9.5 days and $\$ 40,046$ dollars, respectively. Patients that had MRSA compared with non-MRSA cases were noted to have a significantly longer length of hospital stay.

Because no newer agent has shown superiority, vancomycin is commonly employed in the treatment of complicated ABSSSIs and is recommended as first-line therapy in the Infectious Disease Society of America (IDSA) MRSA guidelines and skin and soft tissue infection guidelines when MRSA is suspected [7-9]. Since the last edition of the IDSA MRSA Guidelines in 2011, several new agents have been approved for the treatment of ABSSSIs, including those caused by MRSA. Approval studies for these agents, however, only need to meet the primary end point of non-inferiority based on a pre-defined margin [10]. Although the drug approval trials were designed to prove non-inferiority, ceftaroline demonstrated a more rapid time to resolution of symptoms at day 3 compared with vancomycin in a subset of patients $(n=797)$ with lesion size $\geq 75 \mathrm{~cm}^{2}$ and/or a major abscess $\geq 5 \mathrm{~cm}$. This current study seeks to determine if ceftaroline will result in more rapid resolution of ABSSSIs in a real-life cohort of patients $[11,12]$. The primary purpose of this study was to assess clinical outcomes of ceftaroline versus vancomycin in the treatment of patients hospitalized with complicated ABSSSIs with respect to early clinical outcomes. We additionally estimated the potential economic impact of these two agents by assessing overall costs.

\section{METHODS}

\section{Patient Population and Setting}

This study was conducted at three large academic medical centers within the Detroit metropolitan area: Detroit Medical Center 
(Detroit Receiving Hospital, Harper University Hospital, Sinai-Grace Hospital, Huron Valley Sinai Hospital), Henry Ford Hospital, and St. John's Hospital. Patients were eligible if: 18-89 years of age; presented to the emergency department with complicated ABSSSI defined as wound/surgical site infections, cellulitis, and major abscesses by FDA guidance; required parenteral antibiotics; anticipated to be admitted for at least 2 days, were at risk for MRSA, or had ABSSSI caused by MRSA. Risk factors for MRSA included prior antibiotic use (60 days to presentation), prior hospital exposure (180 days to presentation), presence of skin ulcer; central venous catheters, and known history of MRSA. Patients were excluded if any one of the following was present or suspected: gas gangrene, necrotizing infections, osteomyelitis, complicated bacteremia, endocarditis, and infection due to a gram-negative pathogen or other grampositive pathogen not identified as Staphylococcus aureus or Streptococcus; known resistance to ceftaroline and/or vancomycin, treatment of ABSSSI for $\geq 24 \mathrm{~h}$ before enrollment, surgical incision, and drainage without antibiotics; requirement for renal replacement therapy; life expectancy $<2$ month; burn $>30 \%$ body surface area; known allergy to study drug; pregnant or nursing; or a prisoner at time of randomization. Wayne State Institutional Review Board (114211MP4F), Henry Ford Institutional Review Board (9195), and St. Johns Hospital (IRB \# 383245-17) approved this trial. All procedures performed in studies involving human participants were in accordance with the ethical standards of the institutional and/or national research committee and with the 1964 Helsinki Declaration and its later amendments or comparable ethical standards. Informed consent was obtained from all individual participants included in the study. All study subjects completed the informed consent process.

\section{Experimental Design}

This was a multicenter, prospective, open-label, randomized trial of ceftaroline versus vancomycin for the treatment of ABSSSI. Patients were prospectively identified through clinical surveillance systems (TheraDoc ${ }^{\circledR}$, Salt Lake City, UT, or SENTRI7, Washington, DC) daily based on orders of IV vancomycin, ampicillin-sulbactam, or clindamyci, as these were most likely to have an indication of skin and soft tissue infection. Informed consent was obtained prior to the start of study antibiotic. After consent, patients were randomized (via computerized random mix block generator nQuery Advisor ${ }^{\circledR}$ 7.0) $1: 1$ to ceftaroline \pm metronidazole or to vancomycin \pm ceftriaxone \pm metronidazole or ampicillin/sulbactam by study site. Ceftaroline was dosed according to renal function. Vancomycin was dosed according to pharmacy protocol of each respective institution to a target serum trough concentration of $10-20 \mathrm{mg} / \mathrm{l}$ within the first $72 \mathrm{~h}$. Data were collected using the REDCap electronic data collection system [13].

\section{Clinical Data}

Prospective data collection included size of the lesion $\left(\mathrm{cm}\right.$ or $\left.\mathrm{cm}^{2}\right)$ at time of consent and randomization as well as size of the lesion at follow-up (2-3 days). Information extracted from electronic medical records (eMR) included demographics and baseline characteristics, primary diagnoses, comorbid conditions (Charlson comorbidity index), location within the hospital, and duration of hospitalization and ICU stay. Clinical, laboratory, and radiologic data related to the infection were also collected, including microbiologic cultures, duration of antibacterial therapy, and clinical outcomes such as resolution of fever and white blood cell count. In addition to standard clinical microbiology performed as standard of care, polymerase chain reaction-based rapid diagnostic testing (RDT) was performed on a subgroup of specimens via Cepheid GeneXpert ${ }^{\circledR}$ II MRSA/SA SSTI Assay (Cepheid Inc., Sunnyvale, CA, USA). The agreement between RDT and clinical microbiology S. aureus and MRSA identification was determined. 


\section{Clinical and Economic Definitions and Outcomes}

The primary outcome of interest was to evaluate differences in clinical response at day 2 or 3 of antibiotic therapy between patients receiving vancomycin versus ceftaroline. Clinical response was defined as cessation of spread of lesion (no increase from baseline) and absence of fever at the early assessment time point. Secondary clinical outcomes were number of responders with $\geq 20 \%$ reduction of lesion size at end of day 2 or 3 of study drug and the overall clinical response as noted by the primary treating physician at the end of inpatient treatment. Overall clinical response was defined as cure versus improved versus failure. Cure was resolution of pre-treatment signs and symptoms of infection with no additional need for antibiotic therapy. Improved was defined as incomplete resolution of pretreatment signs and symptoms and/or need for additional antibiotic therapy. Failure was defined as persistent signs and symptoms of infection, a need for change in antibiotic therapy from study drug, and/or need for $>14$ days of antibiotic therapy for ABSSSI. Cost of care was determined by overall length of stay, which was estimated using 2015 American Hospital Association total cost estimates [14].

Analysis of clinical outcomes was completed using the clinically evaluable (CE) patient population. The CE population was defined as all patients who received at least $48 \mathrm{~h}$ of study drug and had follow-up measurement of their lesion. Intention-to-treat (ITT) patients were those initially consented and randomized who received at least one dose of study antibiotic but did not have follow-up measurement of their lesion.

\section{Statistical Analysis}

Assuming a statistical power of $80 \%$, two-sided $\alpha$ of 0.05 , a minimum of $166 \mathrm{CE}$ patients were needed to detect a $20 \%$ difference in primary clinical outcome. Descriptive statistics were reported as percentages, means and standard deviations, or medians with interquartile ranges (IQRs), as applicable. Dichotomous variables were compared using Pearson's chi-squared test or Fisher's exact test, as applicable. Continuous variables were compared using Wilcoxon ranksum test or Student's $t$-test, as applicable. Variables with $p<0.05$ were considered statistically significant. Multivariable backwards-stepwise logistic regression was performed to determine variables independently associated with clinical failure. Variables associated with the primary outcome upon univariate analysis with $p<0.1$ or determined to be clinically relevant a priori were included in the model. The Kaplan-Meier estimator with log-rank statistic was used to determine time to resolution of fever and normalization of WBC between patients treated with vancomycin or ceftaroline. Statistical analysis was performed using IBM SPSS version 22.0 (IBM Corp., Armonk, NY, USA). A post hoc futility analysis was completed to determine the necessary sample size to determine statistically significant differences in the primary clinical outcome (SAS version 9.4, SAS Institute Inc., Cary, NC, USA).

\section{RESULTS}

From April 2012 to May 2016, 174 patients were consented and enrolled; 108 patients met CE criteria. Among the ITT patients, 82 were randomized to receive ceftaroline and 92 to receive vancomycin. Of the CE patients, 54 were randomized to ceftaroline and 54 to vancomycin. The mean age of the entire CE cohort was 51.4 years \pm 15.4 years, there were more males $(58,53.7 \%)$, the majority were African American $(58,53.7 \%)$, and cellulitis was the most common type of ABSSSI $(89,82.4 \%)$, followed by abscess $(30,27.8 \%)$. Overall, the cohort had a low level of comorbidity with a median Charlson score of 3 (IQR 1-5). There were no significant differences in baseline characteristics or measured comorbid conditions with the exception of age; Charlson score was also numerically higher in the ceftaroline arm (Table 1). The majority of patients in both groups were admitted to medical services $(94.1 \%$ vs. $85.7 \%, p=0.11)$. One patient in the ceftaroline group and zero patients in the vancomycin group were admitted to the ICU. A total of $44(40.8 \%)$ patients presented with 
Table 1 Baseline demographics and clinical characteristics of the CE population

\begin{tabular}{|c|c|c|c|}
\hline Characteristic & VAN $(n=54)$ & $\mathrm{CPT}(n=54)$ & $p$ value \\
\hline Mean age, years (SD) & $48.1(16.5)$ & $54.8(13.6)$ & 0.018 \\
\hline Charlson score (IQR) & $2(0-4)$ & $4(1-5)$ & 0.074 \\
\hline Baseline SCr, mg/dl (IQR) & $0.90(0.77-1.10)$ & $0.97(0.72-1.28)$ & 0.758 \\
\hline \multicolumn{4}{|l|}{ Type of ABSSSI } \\
\hline Cellulitis & $43(79.6)$ & $46(85.2)$ & 0.448 \\
\hline Abscess & $16(29.6)$ & $14(25.9)$ & 0.667 \\
\hline Wound infection & $2(3.7)$ & $3(5.6)$ & 0.674 \\
\hline \multicolumn{4}{|l|}{ Site of ABSSSI } \\
\hline Upper extremity & $6(11.1)$ & $11(20.4)$ & 0.186 \\
\hline Lower extremity & $36(66.7)$ & $33(61.1)$ & 0.548 \\
\hline Abdomen & $7(13.0)$ & $3(5.6)$ & 0.184 \\
\hline Chest & $2(3.7)$ & $1(1.9)$ & 0.558 \\
\hline Head/neck & $0(0.0)$ & $1(1.9)$ & 0.315 \\
\hline History of DM without end organ damage, $n(\%)$ & $2(3.7)$ & $2(3.7)$ & 1.000 \\
\hline History of DM with end organ damage, $n(\%)$ & $14(25.9)$ & $15(27.8)$ & 0.828 \\
\hline History of ESLD, $n(\%)$ & $2(3.7)$ & $1(1.9)$ & 0.558 \\
\hline IV drug user, $n(\%)$ & $8(14.8)$ & $4(7.4)$ & 0.221 \\
\hline CKD (not HD) & $4(7.4)$ & $8(14.8)$ & 0.221 \\
\hline History of COPD, $n(\%)$ & $5(9.3)$ & $8(14.8)$ & 0.375 \\
\hline Prosthetic device, $n$ (\%) & $2(3.7)$ & $4(7.4)$ & 0.401 \\
\hline Chronic skin ulcer, $n$ (\%) & $9(16.7)$ & $5(9.3)$ & 0.252 \\
\hline Antibiotics past 30 days, $n$ (\%) & $7(13.0)$ & $7(13.0)$ & 1.000 \\
\hline Prior ABSSSI past 1 year, $n(\%)$ & $7(13.0)$ & $11(20.4)$ & 0.302 \\
\hline Prior hospitalization past year, $n(\%)$ & $19(35.2)$ & $18(33.3)$ & 0.839 \\
\hline Surgery past 30 days, $n$ (\%) & $0(0.0)$ & $2(3.7)$ & 0.153 \\
\hline MRSA infection past year, $n(\%)$ & $1(1.9)$ & $1(1.9)$ & 1.000 \\
\hline
\end{tabular}

$S C r$ serum creatinine, $A B S S S I$ acute bacterial skin and skin structure infection, $D M$ diabetes mellitus, $E S D L$ end-stage liver disease, $I V$ intravenous, $C K D$ chronic kidney disease, $C O P D$ chronic obstructive pulmonary disease

abnormal WBC (35.8\% ceftaroline vs. $46.0 \%$ vancomycin, $p=0.3)$. The median lesion size at time of randomization in the ceftaroline group was $236.0 \mathrm{~cm}^{2}$ (IQR $86.6-790.0 \mathrm{~cm}^{2}$ ) and in the vancomycin group was $275.0 \mathrm{~cm}^{2}$ (IQR $\left.150.0-675.0 \mathrm{~cm}^{2}\right), p=0.62$.
There was no significant difference in primary outcome of day 2 or 3 clinical response $(50.0 \%$ vs. $51.9 \%, p=0.92)$. Follow-up lesion size reduction was not statistically different between patients who received ceftaroline versus vancomycin $[16.7 \%$ (IQR $0-30.8 \%$ ) vs. 
Table 2 Variables associated with clinical failure in multivariable analysis

\begin{tabular}{lllll}
\hline Factor & Unadjusted OR & $\begin{array}{l}\text { 95\% confidence } \\
\text { interval }\end{array}$ & Adjusted OR & $\begin{array}{l}\text { 95\% confidence } \\
\text { interval }\end{array}$ \\
\hline IV drug use & 0.244 & $0.055-1.080$ & 0.251 & $0.054-1.159$ \\
Charlson score & 1.300 & $1.061-1.592$ & 1.305 & $1.065-1.599$ \\
Prior antibiotics & 3.066 & $1.193-7.878$ & 2.807 & $0.946-8.332$ \\
DM with end organ damage & Removed from model & & \\
Vancomycin group & Removed from model & & \\
\hline
\end{tabular}

Hosmer-Lemmeshow $p=0.209$

$15.3 \%$ (IQR $1.7-30.8 \%), p=0.75]$. Of the patients with an abnormal WBC, there was no significant difference in mean time to normalization (2.4 \pm 0.4 days vs. $1.8 \pm 0.2$ days). Additionally, there was no difference in mean time to temperature normalization ( $2.1 \pm 0.4$ days vs. $1.3 \pm 0.1$ days). There was no significant difference in clinical cure/improvement rates at end of ceftaroline or vancomycin inpatient therapy $(81.5 \%$ vs. $75.6 \%$, $p=0.64$ ). Upon univariate analysis history of diabetes with end organ damage, prior antibiotics, IVDU, and Charlson score were associated with treatment failure. Multivariable binary logistic regression was performed with treatment group forced into the model, and only a higher Charlson score was independently associated with clinical failure (Table 2).

The median length of stay in the vancomycin arm was 3.5 days (IQR 3-5 days) versus 4 days (IQR 3-6 days) in the ceftaroline arm, $p=0.43$. The median total cost of care for ceftaroline patients was $\$ 5200$ USD (IQR $\$ 3900-\$ 7800$ ) versus $\$ 4550$ (IQR \$3900-\$6500), $p=0.93$. The majority $(71.3 \%)$ of patients were discharged on oral antibiotics to complete their course of therapy; clindamycin (25.9\%), TMP/ SMX (13.0\%), and cephalexin (12.0\%) were the most common agents used. There was no significant difference in discharge disposition, with $79.6 \%$ of vancomycin-treated and $85.2 \%$ of ceftaroline-treated patients discharged home $(p=0.61)$. Thirty-day infection-related ED revisits and readmissions were also similar between the two treatment groups. Only 9.3\% of vancomycin-treated and $5.6 \%$ of ceftarolinetreated patients were re-admitted within 30 days of their index visit $(p=0.72)$.

In the ITT/CE cohort, 55/44 (ITT/CE) organisms were identified via standard clinical microbiology: 21 patients in the ceftaroline group and 23 patients in the vancomycin group. Among CE patients with culture-positive results, $15(71.4 \%)$ of the isolates in the ceftaroline group and $17(73.9 \%)$ in the vancomycin group were $S$. aureus. Oxacillin resistance was identified in $61.1 \%$ and $85.7 \%$ of these isolates, respectively. The remainder of the organisms identified included coagulasenegative staphylococci (9.1\%), streptococci spp. (11.4\%), and one Escherichia coli and one Pseudomonas aeruginosa. In addition to the standard clinical microbiology, polymerase chain reaction (PCR)-based rapid diagnostic testing to detect MRSA or MSSA was performed on 54 ITT/ CE specimens during the study. When MRSA or MSSA was isolated by clinical microbiology, the RDT was correct in all cases, resulting in 100\% agreement. Among 20 non-S. aureus-positive cultures, the RDT identified $S$. aureus in 4 $(20.0 \%)$. All four of these disagreements were observed in polymicrobial cultures.

A post hoc futility analysis was completed to determine the necessary sample size to detect a statistically significant difference in primary outcome given the observed difference of $1.9 \%$. To obtain $p<0.05,10,989$ patients needed to be clinically evaluable in each treatment arm (Table 3). 
Table 3 Results of post-hoc futility analysis

\begin{tabular}{lccll}
\hline Futility analysis & Per group sample size & Total sample size & Lower bound nominal $\boldsymbol{p}$ & Significance threshold \\
\hline 1 & 1558 & 3116 & 0.0758 & 0.92 \\
2 & 3115 & 6230 & 0.1955 & 0.80 \\
3 & 4672 & 9344 & 0.3565 & 0.64 \\
4 & 6229 & 12,458 & 0.5295 & 0.47 \\
5 & 7786 & 15,572 & 0.6872 & 0.31 \\
6 & 9343 & 18,686 & 0.8122 & 0.18 \\
7 & 10,900 & 21,800 & 0.8998 & 0.1 \\
8 & 12,457 & 24,914 & 0.9578 & 0.04 \\
\hline
\end{tabular}

Need $n=10,989$ per group to achieve a $1.9 \%$ difference in primary outcome with $80 \%$ power at $\alpha=0.05$

\section{DISCUSSION}

This was a prospective multicenter study evaluating the impact of ceftaroline versus vancomycin for the treatment of patients hospitalized with ABSSSIs. Over a 4-year period, we were unable to achieve our initial sample size of 166 CE patients. One major hindrance to meeting the a priori sample size was the changing practices for the management of ABSSSIs, with treatment moving increasingly to the outpatient setting [3]. For instance, based on internal data, treatment of ABSSSIs that initially required hospitalization often resulted in lengths of stay of 3-5 days, making early test-ofcure analysis challenging. In a previous clinical evaluation of ABSSSIs in hospitalized patients at the Detroit Medical Center (DMC), our group demonstrated improved efficacy and decreased costs when daptomycin was compared with vancomycin [15]. Daptomycin achieved more rapid resolution of infection, a greater overall clinical cure, shorter mean length of stay (4 vs. 7 days, $p<0.001$ ) compared with vancomycin. This difference resulted in a significant $(\$ 5027$ vs. $\$ 7553, p<0.001)$ median cost difference favoring daptomycin. This study, however, was conducted a decade ago. In a more recent pragmatic study comparing vancomycin to daptomycin for the treatment of ABSSSIs, Kauf et al. found no significant difference in clinical success [16]. The likelihood day-2 response was lower in the vancomycin arm through multivariable regression analysis; this result, however, demonstrated a low degree of precision and $95 \%$ CI close to 1.0 (OR $=0.498,95 \%$ CI 0.249-0.997). Our results demonstrated similar day 2 and/or 3 clinical responses between vancomycin and ceftaroline and similar overall patient outcomes requiring a sample size > 20,000 patients to detect a statistically significant, though arguably not clinically significant, outcome.

During this study, we also had the opportunity to evaluate a polymerase chain reactionbased RDT platform on a subgroup of patients [17]. The results demonstrate consistent ability to detect $S$. aureus and MRSA when present and are consistent with other evaluations of this technology demonstrating high sensitivity. Given the high prevalence of MRSA among ABSSSIs, the improvement in time to identification via RDT has the potential to rapidly streamline antibiotic therapy to improve time to optimal therapy and minimize unnecessarily broad-spectrum coverage. We did, however, experience difficulties in using RDTs to assist in enrolling patients with specific pathogens, in particular the limited samples that could be run in a timely manner. Few patients had viable samples for testing, and timing of collection may be challenging if the infection was incised and drained prior to screening. Nonetheless, the technology demonstrates the potential to assist 
studies in targeting certain patient populations based on microorganisms identified.

Several limitations need to be addressed. Although the study was randomized, there were several imbalances in the patient cohorts. In particular, patients treated with ceftaroline had higher Charlson Comorbidity Index scores, higher rates of $\mathrm{CKD}$, higher rates of prosthetic involvement, and higher rates of previous ABSSSIs. Although individually these differences were not statistically significant, taken together they may account for the trend toward improved outcomes in vancomycin therapy; however, the sample size is too small for formal assessment. Additionally, the study was heavily weighted toward the treatment of cellulitis as opposed to wounds or abscesses. Although patients were required to have risk factors for MRSA to be enrolled in the study, it is still likely that the majority of these infections were caused by streptococci and not $S$. aureus. Importantly, $55 \%$ of patients included in the analysis demonstrated response to therapy after 1 day of antibiotics. This finding, coupled with the fact that patients could receive up to $24 \mathrm{~h}$ of therapy before enrollment, also helps to explain our findings. Lastly, due to the nature of the patient population under study, of which many frequently visit multiple healthcare facilities in the metro Detroit area, there is the potential to have missed ED visits or hospitalizations to facilities outside those included in this study.

Another important consideration is based on published data demonstrating that early clinical response is influenced by more than antibiotic selection [18]. In a recently published study from Bruun et al., day 2 or 3 response in ABSSSIs occurred commonly (90\% of patients) and was related to antibiotic therapy, but also other non-pharmacologic factors such as type of ABSSSI, duration of symptoms before treatment, and BMI. The authors recommended a combined clinical and biochemical parameter for response to therapy. In fact, the results of the DISCOVER trial have been used to highlight the lack of clinical relevance of this end point for determining successful outcomes [19]. This is not without controversy, however [20]. A recently published pooled analysis of ESTABLISH trials reported a high predictive value for clinical cure $[20,21]$. Although it is recommended that sustained clinical response through continued resolution of signs and symptoms of infection 7-14 days after therapy should be measured, the patient population of the Detroit metropolitan area made this followup assessment challenging [22].

\section{CONCLUSION}

Early clinical response between vancomycinand ceftaroline-treated ABSSSIs was similar in this open-label randomized clinical trial. The majority of patients in this study presented with cellulitis and were allowed to have up to $24 \mathrm{~h}$ of antibiotic therapy before enrollment, which may limit generalizability of the current results. Additionally, patients with ABSSSIs rarely remained hospitalized for $>2-3$ days, thus limiting our ability to critically assess clinical outcomes.

\section{ACKNOWLEDGEMENTS}

Thank you to the participants of this study for their cooperation and willingness to help advance clinical trial data in the management of ABSSSIs. Special thanks to Hyunuk Seung, Biostatistician at University of Maryland School of Pharmacy, Department of Pharmacy Practice, for assistance with the futility analysis.

Funding. This was an investigator-initiated study funded by Allergan plc. No article processing charges were received by the journal for the publication of this article. All authors had full access to all of the data in this study and take complete responsibility for the integrity of the data and accuracy of the data analysis.

Authorship. All named authors meet the International Committee of Medical Journal Editors (ICMJE) criteria for authorship for this article, take responsibility for the integrity of the work as a whole, and have given their approval for this version to be published. 
Disclosures. Susan Davis has served on advisory boards for Achaogen, Nabriva, and Melinta. Michael Rybak has received grant support, participated on speaker bureaus or consulted for Accelerate, Achaogen, Allergan, Bayer, Merck, and Shionogi, and was partially supported by NIAIDNIAID R01 AI121400. Michael Rybak is also Editor in Chief of Infectious Diseases and Therapy. Kimberly Claeys serves on the advisory board for Melinta Therapeutics and Nabriva. Keith Kaye is a consultant for Allergan. Donald Levine previously received grant support from Allergan, Melinta, and NIHAI2008025 and participated on speaker bureaus or consulted for Allergan, Merck, Novartis, and Contrafect. Jason Pogue was a speaker for Allergan, Merck, and Melinta and received grant support from Merck. Anthony Casapao received grant support from Allergan, Merck, and Astellas Pharmaceuticals and served as a consultant with Allergan and Shionogi and on the advisory boards for Melinta, Paratek, Tetraphase, and Nabriva. Evan Zasowski, Trang Trinh, Suprat Wilson, Kate Reyes, Pamela Hartman, James Gordon, Christopher Giuliano, Robert Takla, Colleen Rieck, Leonard Johnson, George Delgado, Crystral Arthur, Nitin Bhatia, Ryan Mynatt, Robert Welch, Robert Sherwin, Wasif Hafeez, and Kyle Murray have nothing to disclose. At the time of the study, both Evan Zasowski and Trang Trinh were affiliated with Anti-Infective Research Laboratory, Wayne State University Eugene Applebaum College of Pharmacy and Health Sciences, Detroit, MI, USA. Evan Zasowski's current affiliation is Department of Pharmaceutical Sciences, Touro University, Vallejo, CA, USA. Trang Trinh's current affiliation is Department of Clinical Pharmacy, University of California San Francisco, San Francisco, CA, USA.

Compliance with Ethics Guidelines. Wayne State Institutional Review Board (114211MP4F), Henry Ford Institutional Review Board (9195), and St. Johns Hospital (IRB \# 383245-17) approved this protocol. All procedures performed in studies involving human participants were in accordance with the ethical standards of the institutional and/or national research committee and with the 1964 Helsinki Declaration and its later amendments or comparable ethical standards. Informed consent was obtained from all individual participants included in the study.

Data Availability. The data sets generated during and/or analyzed during the current study are not publicly available due ownership by Allergan plc, but are available from the corresponding author on reasonable request.

Open Access. This article is distributed under the terms of the Creative Commons Attribution-NonCommercial 4.0 International License (http://creativecommons.org/licenses/ by-nc/4.0/), which permits any noncommercial use, distribution, and reproduction in any medium, provided you give appropriate credit to the original author(s) and the source, provide a link to the Creative Commons license, and indicate if changes were made.

\section{REFERENCES}

1. Dryden MS. Complicated skin and soft tissue infection. J Antimicrob Chemother. 2010;65(Suppl 3):iii35-44.

2. Edelsberg J, Taneja C, Zervos M, Haque N, Moore C, Reyes K, et al. Trends in US hospital admissions for skin and soft tissue infections. Emerg Infect Dis. 2009;15(9):1516-8.

3. Pollack CV, Amin A, Ford WT, Finley R, Kaye KS, Nguyen $\mathrm{HH}$, et al. Acute bacterial skin and skin structure infections (ABSSSI): practice guidelines for management and care transitions in the emergency department and hospital. J Emerg Med. 2015;48(4):508-19.

4. Hersh AL, Chambers HF, Maselli JH, Gonzales R. National trends in ambulatory visits and antibiotic prescribing for skin and soft-tissue infections. Arch Intern Med. 2008;168(14):1585-91.

5. Kaye KS, Patel DA, Stephens JM, Khachatryan A, Patel A, Johnson K. Rising United States hospital admissions for acute bacterial skin and skin structure infections: recent trends and economic impact. PLoS One. 2015;10(11):e0143276.

6. Itani KMF, Merchant S, Lin S-J, Akhras K, Alandete JC, Hatoum HT. Outcomes and management costs 
in patients hospitalized for skin and skin-structure infections. Am J Infect Control. 2011;39(1):42-9.

7. Liu VX, Fielding-Singh V, Greene JD, Baker JM, Iwashyna TJ, Bhattacharya J, et al. The timing of early antibiotics and hospital mortality in sepsis. Am J Respir Crit Care Med. 2017;196(7):856-63.

8. Stevens DL, Bisno AL, Chambers HF, Dellinger EP, Goldstein EJC, Gorbach SL, et al. Practice guidelines for the diagnosis and management of skin and soft tissue infections: 2014 update by the Infectious Diseases Society of America. Clin Infect Dis. 2014;59(2):147-59.

9. Stevens DL, Bisno AL, Chambers HF, Everett ED, Dellinger P, Goldstein EJC, et al. Practice guidelines for the diagnosis and management of skin and softtissue infections. Clin Infect Dis. 2005;41(10):1373-406.

10. Food and Drug Administration (FDA). Non-inferiority clinical trials to establish effectiveness guidance for industry. https://www.fda.gov/downloads/ Drugs/Guidances/UCM202140.pdf [cited 2019 Jan 10].

11. Wilcox MH, Corey GR, Talbot GH, Thye D, Friedland D, Baculik T, et al. CANVAS 2: the second Phase III, randomized, double-blind study evaluating ceftaroline fosamil for the treatment of patients with complicated skin and skin structure infections. J Antimicrob Chemother. 2010;65(Suppl 4):iv53-65.

12. Corey GR, Wilcox MH, Talbot GH, Thye D, Friedland D, Baculik T. CANVAS 1: the first Phase III, randomized, double-blind study evaluating ceftaroline fosamil for the treatment of patients with complicated skin and skin structure infections. J Antimicrob Chemother. 2010;65(Suppl 4):iv41-51.

13. Harris PA, Taylor R, Thielke R, Payne J, Gonzalez N, Conde JG. Research electronic data capture (REDCap)-a metadata-driven methodology and workflow process for providing translational research informatics support. J Biomed Inform. 2009;42(2):377-81.

14. AHA Annual Survey | American Hospital Association's Annual Survey Database | AHA Data Online [Internet]. [cited 2019 Jan 10]. https://www. ahadataviewer.com/additional-data-products/AHASurvey/.
15. Davis SL, McKinnon PS, Hall LM, Delgado G, Rose $\mathrm{W}$, Wilson RF, et al. Daptomycin versus vancomycin for complicated skin and skin structure infections: clinical and economic outcomes. Pharmacotherapy. 2007;27(12):1611-8.

16. Kauf TL, McKinnon P, Corey GR, Bedolla J, Riska PF, Sims M, et al. An open-label, pragmatic, randomized controlled clinical trial to evaluate the comparative effectiveness of daptomycin versus vancomycin for the treatment of complicated skin and skin structure infection. BMC Infect Dis. 2015;15:503.

17. Wolk DM, Struelens MJ, Pancholi P, Davis T, DellaLatta P, Fuller D, et al. Rapid detection of Staphylococcus aureus and methicillin-resistant $S$. aureus (MRSA) in wound specimens and blood cultures: multicenter preclinical evaluation of the Cepheid Xpert MRSA/SA skin and soft tissue and blood culture assays. J Clin Microbiol. 2009;47(3):823-6.

18. Bruun T, Oppegaard O, Hufthammer KO, Langeland $\mathrm{N}$, Skrede S. Early response in cellulitis: a prospective study of dynamics and predictors. Clin Infect Dis. 2016;63(8):1034-41.

19. Dunne M. Abstract: concordance of clinical response at 48-72 hours after initiation of therapy and end of treatment (EOT) in patients with acute bacterial skin and skin structure infection (abSSSI) in the DISCOVER Studies (IDWeek 2013) [Internet]. Poster presented at: IDWeek 2013; 2013 [cited 2019 Jan 10]; San Fransisco. https://idsa.confex.com/ idsa/2013/webprogram/Paper42449.html.

20. Nathwani D, Dryden M, Garau J. Early clinical assessment of response to treatment of skin and soft-tissue infections: how can it help clinicians? Perspectives from Europe. Int J Antimicrob Agents. 2016;48(2):127-36.

21. Nathwani D, Corey R, Das AF, Sandison T, De Anda C, Prokocimer P. Early clinical response as a predictor of late treatment success in patients with acute bacterial skin and skin structure infections: retrospective analysis of 2 randomized controlled trials. Clin Infect Dis. 2017;64(2):214-7.

22. Food and Drug Administration (FDA). Guidance for industry acute bacterial skin and skin structure infections: developing drugs for treatment. https:// www.fda.gov/downloads/Drugs/Guidances/ucm071 185.pdf [cited 2019 Jan 10]. 